UMS/HEP 95-002

submitted to NIM

9 October 1995

\title{
A Simple Multiprocessor Management System for Event-Parallel Computing
}

\author{
Steve Bracker, Krishnaswamy Gounder, Kevin Hendrix, and Don Summers \\ Department of Physics and Astronomy \\ University of Mississippi-Oxford \\ University, MS 38677 USA
}

\begin{abstract}
Offline software using TCP/IP sockets to distribute particle physics events to multiple UNIX/RISC workstations is described. A modular, building block approach was taken, which allowed tailoring to solve specific tasks efficiently and simply as they arose. The modest, initial cost was having to learn about sockets for interprocess communication. This multiprocessor management software has been used to control the reconstruction of eight billion raw data events from Fermilab Experiment E791.
\end{abstract}

\section{The E791 Reconstruction Task}

Fermilab Experiment E791 accumulated a large dataset (50 Terabytes, 20 billion events, $240008 \mathrm{~mm}$ Exabyte tapes) in 1991 and early 1992 [1,2]. As might be expected, the reconstruction and analysis of this data challenged available computing resources; event reconstruction alone required over 10000 mips-years of processing power. For $2 \frac{1}{2}$ years, reconstruction processing was underway at four different locations [3]. The three largest sites used clusters or farms of commercial UNIX/RISC workstations connected together by thin-wire Ethernet. Within each farm, many processors operated together; data management and system control were exerted from a single point via multiprocessor management software. Here we describe the multiprocessor management software developed and used at the University of Mississippi [4]. The Mississippi farm hardware is shown in Figs. 1 and 2.

Most of the large-scale computing needs encountered in particle physics - reconstruction and analysis of recorded data and generation of simulated data - are event-oriented. Each event's data packet is extracted from an input stream and processed in isolation from other events. The results from each event's analysis are merged into an output stream. The computing power required to process each data packet is significant relative to the time needed to transport the data, even over datapaths of modest throughput. Many other scientific computing problems (e.g. small-object recognition in astronomical images) conform to the same model. In all such problems, it is trivial to divide the total computing task over many processors; each processor is simply given a share of the events to process independently of its peers.

Most reconstruction and simulation tasks are developed and tested as single-processor programs. They can be outlined in the following manner:

initialize data structures

initialize input and output data streams

read run startup information from input data stream

read and store run calibration constants

for every event from the input stream...

unpack and check the input data

PROCESS THE EVENT (most of the work)

for good events,

pack the processed event into output stream

produce a report characterizing the processing job stop 
When the program is "nearly working," it is then moved into the multiprocessing environment for final testing and production. If it has been written in a sensible fashion, with dataflow management tasks cleanly separated from event processing tasks, then the transition from single processor to multiprocessor system should be relatively painless. Dataflow management is generally vested in a single server process, while the event processing is performed by numerous client processes running in many processors. The server grants the clients access to calibration data, reads the event input stream, parcels out events to the many client processors, gathers output data from the clients, and writes the output stream. The server processor is typically well endowed with peripheral devices (disks, tape drives), whereas the clients may be very simple (but powerful) processors with no peripherals except a network connection.

\section{The Multiprocessor Manager's Tasks}

In the first implementations of event-oriented multiprocessing for particle physics $[5,6]$, the multiprocessor management software had to do a great deal of hard work. Clients frequently had no significant operating system, primarily because memory was expensive. Executable code and calibration data had to be formatted in the server and downloaded into the clients word by word. Extraction of input events from records and merging of output events into records often had to be done in the server because of limited client buffer memory, posing the danger of a processing bottleneck at the server. Reports had to be retrieved from the clients as tables of numbers, to be formatted and output by the server. Substantial effort was often required to move a single-processor program onto a multiprocessor system, because the division between server code and client code was intricate. Developing the multiprocessor management code could become nearly as large a task as developing the event processing software: an odious burden.

With the advent of cheaper memory (enabling each client to have a real operating system and large event buffers) plus good networking software, most of the difficulties inherent in moving code to a multiprocessor vanished. Rather than relating "war stories" of heroic efforts and brilliant strategies leading to victory in the face of staggering difficulties, this paper celebrates the fact that multiprocessor management is now straightforward, that an effort tiny compared to the development of application code will suffice to distribute the workload efficiently and reliably among many processors, and that generic system software nearing completion promises to make the task even easier in the future.

\section{A Disk-Based Multiprocessor Manager}

It is easiest to understand the multiprocessor strategy by considering first a disk-based system. The clients each have a real operating system (in our case ULTRIX, a flavor of UNIX) and thus can read and write disk files, but they have no disks attached directly to them. Instead, clients have access over the network to disks attached to the server. Using Network File System (NFS) software, disks or portions of disks can be "cross-mounted" so that they are accessible by multiple processors. For us, this meant the server itself and all of its clients. NFS is supplied as standard software with many types of workstations, and is available as an option on most of the rest.

The fact that clients, though diskless, have full access to disk services across the network, immediately solves several problems that burdened earlier systems:

(1) Clients can read executable code and start programs; it is no longer necessary for the server to micromanage the downloading and startup of clients, though high level control of client processes is still maintained in the server,

(2) Clients can read their own calibration files; it is no longer necessary for the server to explicitly read, reformat, and transmit calibration data for the clients,

(3) Clients can write their own report files; it is no longer necessary for the server to explicitly extract, format, and write reports for the clients.

In many cases, cross-mounted disks can even be used to provide for the movement of event data (input and output) between server and clients: 
(4) The server can read events from tape and write them to disk; the clients can read the events from disk and process them. For example, each client can own two input files; the server inserts events into one while the client consumes events from the other,

(5) The clients can write output events to disk; the server can read the disk files, and write the events to output tapes. Each client can own two output files; the client inserts processed events into one while the server removes events from the other and writes them to tape.

Referring back to the outline of the processing tasks, it is clear that the program running in each client of a multiprocessor system is very similar to the single-processor program. Each client loads its own code and starts it. Each client reads its own calibration constants from disk. Each client writes its own reports to disk. Each client reads its input data from disk and writes its output data to disk, just as single-processor code usually does during program development. The server's tasks now become the following:

(1) Keeping a list of the available processors and requesting them to start instances of the processing program,

(2) Making startup data (e.g. run number) available to clients in a disk file,

(3) Assuring that valid calibration constants are available to be read by the clients,

(4) Reading input data from tape and distributing events to the various client disk files,

(5) Fetching output data from the various client disk files and writing it to tape,

(6) Gathering reports from the client report files and producing (where appropriate) a system-wide report.

This scheme was very simple to implement, maintain, and document. For applications in which dataflow is slow, it works very well. Clients are provided with certified events (or blocks of events) on a reliable medium, protected from the vagaries of tape reading, which is handled by the server. Likewise, clients have a reliable medium for writing output; problems with tape writing are handled in the server. If enough disk is available, whole tape files can be staged through the disk files, leading to more reliable performance from streaming tape drives [7].

\section{A TCP/IP Sockets Based Multiprocessor Manager}

As a farm's data throughput is increased by adding processors, upgrading processors, or making application code run faster, passing the event stream through disk can become a bottleneck; clients may spend too much time waiting for input events to be read and output events to be written, and disk "thrashing" (incessant head motion) may set in. One possible solution is to move the event streams through cross-mounted memory disks using special software by which a portion of the server's memory is set aside and appears to the user as a very high-speed, low-latency, non-thrashing, disk drive. When we explored this option in 1992, such software was available but did not perform well; it is possible that suitable products now exist. Memory disks preserve the advantage that the application code for a multiprocessor system looks almost identical to that for a single processor; all data passing is done with normal Fortran reads and writes.

Instead, our multiprocessor manager bypasses disk altogether for those portions of the dataflow that are fast enough to challenge the disk's throughput; data is moved between processes using TCP/IP (Transmission Control Protocol/Internet Protocol) network services [8]. Using this facility, processes can establish a connection between themselves and pass data back and forth by read_from_connection and write_through_connection subroutine calls. Event input/output can no longer be implemented as simple Fortran reads and writes in the client (a disadvantage), but on the other hand, the high throughput of direct network data transfers becomes available.

Before deciding to implement a TCP/IP-based scheme, we had to answer three significant questions: 
(1) Would passing data through TCP/IP yield a significant improvement in throughput over NFS disk-based data transfers?

(2) Would resulting modifications to the client code be tractable?

(3) Given very limited time to produce the management code, would TCP/IP be easy enough to learn and use, especially for those of us coming from a VAX/VMS - Fortran environment?

We wrote a test package to shuttle data between several processors to measure throughput and reliability. The results: average data throughputs in excess of 900 kilobytes per second (almost full Ethernet speed) could be maintained (we needed only 150 kilobytes per second); the impact on client efficiency was immeasurably low at maximum projected throughput; and data corruption was not seen.

In keeping with the Fortran orientation of the experiment's software, Fortran-callable functions were written in $\mathrm{C}$ for all of the system services needed to support TCP/IP data transfers. As a result, the changes to the client code were minimal and easily understandable to Fortran-only programmers.

Fig. 3 illustrates how the network I/O calls are used. As the server prepares to start a client, it uses make_socket to "have a phone put in", so that it will be able to connect to the client. When the client starts, it too uses make_socket to "have a phone put in". The server "lists its number" by binding its socket to a port (bind_socket), and "stays near the phone" listening for an attempt to connect (listen_socket). The client "calls up" the server (connect_socket) and the server "picks up the phone" establishing the connection (accept_socket).

When it needs input data, the client "places its order" by writing a message to the server (write_socket). The server is continually monitoring all of the client connections for requests (select_socket). When a request comes in, the server "writes down the order" (read_socket), and does its best to satisfy the client's request. The client and server shuttle messages back and forth (each writing to the other and reading from the other) until the input data is exhausted. At that point, the server notifies the clients that there is no more data. The clients then finish their tasks, close their connections (close_socket), and exit; the server finishes its tasks and exits.

The Fortran-callable routines that manipulate sockets and connections really are that simple to use. Only one routine (read_socket) is any more than a C to Fortran interface, and even read_socket is trivial. Almost all of the real work involved with network communications has already been done in UNIX, TCP/IP, and Berkeley Sockets.

\section{Performance}

Only the transport of input events from server to clients was implemented in TCP/IP; output events from clients to server needed to be staged to disk to make best use of our Exabyte tape drives, so they were written to disk through NFS as before. This output scheme was efficient because five out of six events were rejected by a filter after reconstruction and were not output.

Tape reading was multiply buffered, so that events were almost always available immediately when a client requested them. We used a simple trick to ensure that clients were not suffering from delays in receiving new events. Whenever input data was available, the server checked the clients to see which ones were asking for new input. The server always checked the clients in the same order, so processors at the start of the list had priority over processors near the end of the list. If clients were "spinning," i.e. waiting for events, this was reflected in anomalously low throughput in the least favored processors. By this and several additional measures, it appears that more than $97 \%$ of the client cycles were being put to beneficial use (actually processing events) whenever the farm was running at all.

Funding awarded in June 1993 allowed an expansion of the Mississippi computing facility from 1100 to 2900 mips. By July 1993, the increased computing power had been acquired and was processing data. E791 reconstruction was completed in September 1994. A total of eight billion events on 10000 raw data tapes 
were processed at the University of Mississippi. Preliminary versions of the reconstruction software were run to find out which E791 algorithms would yield the most physics per tape. One result was a tripling in the yield of charm particles. When the final reconstruction software was ready, it was run. Overall efficiency, considering all cycles lost for any reason, exceeded 90\%. Thus the multiprocessor manager has proven itself to be efficient and robust under varying conditions in what we believe to be a fairly typical university operating environment.

For the E791 experiment as a whole, the successful management of multiple processors has provided the full reconstruction of over 200000 particles with a charm quark. This large charm sample is in turn generating new physics results [9-15].

\section{Scavenging Computing Cycles}

Although most of the processors in the Mississippi system are mounted in racks at a central location, some are at people's desks and serve as general purpose workstations. Many workstation activities - editing, compiling and running small programs, reading and writing e-mail, etc. - can coexist with the farm client process running in the background. However, there are some workstation activities that are incompatible with farm operations, and the client process must be removed from the workstation. Nevertheless, it is certainly helpful to be able to scavenge the desk workstation cycles when they are otherwise unused.

There are several approaches to using the desk workstations as farm clients. On one extreme, the workstation can be removed from the farm client list; it will never be used as a farm client. At the other extreme, one can forbid workstation activities that interfere with the farm operation. In between there is ample middle ground. For example, it is possible to have the farm server examine the workstation activities from time to time and adjust the priority or run status of the farm client process accordingly.

At Mississippi, we have found it satisfactory to allow users to abruptly kill the client process whenever they find its activities on their workstations to be troublesome. The server is quickly aware that the client has disappeared and adjusts its event distribution accordingly. If a workstation user knows that he will be engaged in a computationally intensive activity for a long time, he may edit the client list and remove his workstation from the farm. Once killed, a workstation cannot participate as a farm client again until the next job is started. Though crude, this scheme has proven highly effective in our operating environment.

One disadvantage of this approach is that a few input events may be "trapped" in a killed processor, and thus lost. We take a rather cavalier attitude toward such losses; with twenty billion events, we can afford to lose a few now and again. Although in principle the server can hold event buffers until successful processing is assured, and reassign the events to another processor if they become trapped in a disabled client, we don't do that. In E791, processing raw events is rather like hauling corn to market in a truck. If a few grains of corn fall out of the truck, no one becomes concerned until the loss becomes large enough to be economically important. The alternative approach - treating events as babies in a hospital nursery, where one normally expects a somewhat stricter accounting - only makes sense when the events have become greatly enriched in scientific significance, late in the reconstruction and analysis cycle.

\section{Multiprocessor Operations}

The most vexing operational problems are those that one might expect in handling a dataset this large ensuring that all tapes are processed exactly once, preparing and maintaining run-dependent calibration files, making sure that all of the output tapes are correctly labeled, preparing and examining the necessary report files, etc. During the first year of operation, job flow was controlled by scripts composed with the help of small interactive programs. In 1993, a farm job manager with an X Window graphical user interface was written. It was specifically targeted at preventing errors we had observed to occur in the setup of jobs and the management of tapes. Most of the day-to-day system operation in later stages was performed by students, many of whom had little understanding of the internal workings of the system.

Farm management software consists of three independent programs:

(1) The server code, which provides system and dataflow control, 
(2) The client code, linked to the application code routines,

(3) The tape-writing code, running on the server, which fetches events written to disk by clients and transfers them to tape.

The server, the tape writer, and each client produces report files for each job. The tape writer (the last program to handle the data) gathers these reports together and produces two farm-wide reports. One is a standard statistics file common to all E791 computing sites; the other is a report tailored to the needs of the Mississippi site.

\section{Future Directions}

Event-parallel computing farms have come to dominate large-scale computations in experimental particle physics. Because computing paradigms seldom remain viable for more than a decade or so in this field, it is perhaps useful to ask "Whither computer farming?" in the next few years.

Two trends in the computer industry make it unlikely that "farming as usual" will continue much longer. First, individual workstations, PCs, and Macintoshes now coming to market offer such prodigious computing power that each processor can exploit the full I/O bandwidth of its data storage peripherals; in that case, there seems little point in concentrating dataflow through a server, although one might still imagine a single locus of system control for several computers, each directly attached to its own peripheral devices [16].

Second, with the increased popularity of object-oriented design and languages such as $\mathrm{C}++$ which support it, the data structures now being explicitly passed between processes will be implemented as objects of classes. Very soon it will be possible to define remote objects, which will tie together the resources of many processors within a single programming environment. For programs written within such a paradigm, there will be almost no difference between a single processor implementation and a multiprocessor implementation except for listing the computing resources that may be brought to bear on the task.

In the early days of "computer farming" in particle physics, there were not suitable commercial processors available, so we built our own $[5,6]$. After a short time, we were put out of the processor building business by high-powered workstations offered by several vendors [17]. The early implementations of multiprocessor management software were complex, costly, and cumbersome to use; the need for them has been snuffed out by the widespread availability of interprocess communication tools such as TCP/IP sockets and NFS. The simple streamlined multiprocessor management toolkits remain and are likely to be in use for a bit longer, but their end is also in sight. For tasks with mammoth computational needs and modest dataflow rates, truly transparent, vendor-independent, object-oriented access to the combined power of dozens or hundreds of inexpensive powerful processors appears to be imminent; "computer farm management" as we now practice it, is an idea whose time has come and nearly gone.

\section{Acknowledgements}

We especially thank Lucien Cremaldi and Breese Quinn for their contributions to building and running the Mississippi farm. This effort was partially supported by the United States Department of Energy, DE-FG05-91ER40622.

\section{References}

[1] D.J. Summers et al., Charm Physics at Fermilab E791, Proceedings of the XXVII ${ }^{\text {th }}$ Recontre de Moriond, Electroweak Interactions and Unified Theories, Les Arcs, France (15-22 March 1992) 417; presents an overview of the experiment.

[2] S. Amato, J.R.T. de Mello Neto, J. de Miranda, C. James, D.J. Summers, and S.B. Bracker, The E791 Parallel Architecture Data Acquisition System, Nucl. Instr. and Meth. A324 (1993) 535; describes the system used to collect the E791 dataset. 
[3] The Ohio State University (moved to Kansas State University in 1993); Fermi National Accelerator Laboratory; Centro Brasileiro de Pesquisas Físicas-Rio de Janeiro; the University of Mississippi.

[4] Although all four processing centers ran the same physics reconstruction code, three different multiprocessor management systems were used.

[5] Paul F. Kunz et al., Experience Using the 168/E Microprocessor for Off-line Data Analysis, IEEE Trans. Nucl. Sci. 27 (1980) 582.

[6] J. Biel et al., Software for the ACP Multiprocessor System, Proc. of the Intl. Conf. on Comp. in High Energy Physics, Asilomar (2-6 Feb. 1987), Comp. Phys. Comm. 45 (1987) 331; FERMILAB-Conf$87 / 22$.

[7] Exabyte tape drives operate most reliably if they read and write data continuously rather than starting and stopping incessantly. In addition, head wear depends on the time the tape is tensioned over the rotating head, not the amount of data written. You obtain far more useful life (Terabytes per head change) from an Exabyte that reads and writes at nearly full speed.

[8] Sidnie Feit, TCP/IP: Architecture, Protocols, and Implementation, McGraw-Hill, Inc. (1993), ISBN 0-07-020346-6, Chapter 17. There is also an extensive bibliography.

[9] A.K.S. Santha, Study of the Decay $D^{0} \rightarrow K_{\mathrm{S}}^{0} K^{ \pm} \pi^{\mp}$, APS/AAPT Joint Meeting, Crystal City, VA (18-22 April 1994); Bull. Amer. Phys. Soc. 39 (1994) 1030.

[10] Tom Carter, Production Asymmetries in $x_{f}$ and $P_{t}^{2}$ for $D^{ \pm}$Mesons, Proc. of the 8th Meeting, Div. of Particles and Fields of the Amer. Phys. Soc., Albuquerque, NM (2-6 August 1994) 513.

[11] Milind Purohit and James Wiener, Preliminary Results on the Decays $D^{+} \rightarrow K^{+} \pi^{+} \pi^{-}, D^{+} \rightarrow$ $K^{+} K^{+} K^{-}$, Proc. of the 8th Meeting, Div. of Particles and Fields of the Amer. Phys. Soc., Albuquerque, NM (2-6 August 1994) 969.

[12] Guy Blaylock, A Search for $D^{0} \bar{D}^{0}$ Mixing at the Fermilab Er91 Experiment, Proc. of the 8th Meeting, Div. of Particles and Fields of the Amer. Phys. Soc., Albuquerque, NM (2-6 August 1994) 980.

[13] Krishnaswamy Gounder and Lucien Cremaldi, D-Meson-Pion Production Correlations, APS/AAPT Joint Meeting, Washington, DC (18-21 April 1995); Bull. Amer. Phys. Soc. 40 (1995) 1022.

[14] Arun Tripathi, Search for $D^{0}-\overline{D^{0}}$ Mixing in Semileptonic Decays, APS/AAPT Joint Meeting, Washington, DC (18-21 April 1995); Bull. Amer. Phys. Soc. 40 (1995) 1035.

[15] E.M. Aitala et al., Search for the Flavor-Changing Neutral Current Decays $D^{+} \rightarrow \pi^{+} \mu^{+} \mu^{-}$and $D^{+} \rightarrow$ $\pi^{+} e^{+} e^{-}$, KSU HEP-95-01, FERMILAB Pub-95/142-E (submitted to Phys. Rev. Lett.).

[16] One caveat. I/O is getting faster but for now not as quickly as CPUs. We used Ethernet at $10 \mathrm{Mbit} / \mathrm{s}$; and Exabyte 8200 and 8205 tape drives storing 2 Gigabytes at 0.25 Mbytes/s. Full Duplex Fast Ethernet now runs at $2 \times 100 \mathrm{Mbits} / \mathrm{s}$. The Quantum DLT4000 tape drive now stores 20 Gigabytes at $1.5 \mathrm{Mbytes} / \mathrm{s}$. The Exabyte Mammoth Tape drive (still in development) is designed to store 20 Gigabytes at 3 Mbytes/s. The current Exabyte 8505XL stores 7 Gigabytes at 0.5 Mbytes/s. Unhappily, the cost of tape per Terabyte stored has not decreased.

[17] C. Stoughton and D.J. Summers, Using Multiple RISC CPUs in Parallel to Study Charm Quarks, Computers in Physics 6 (1992) 371. 


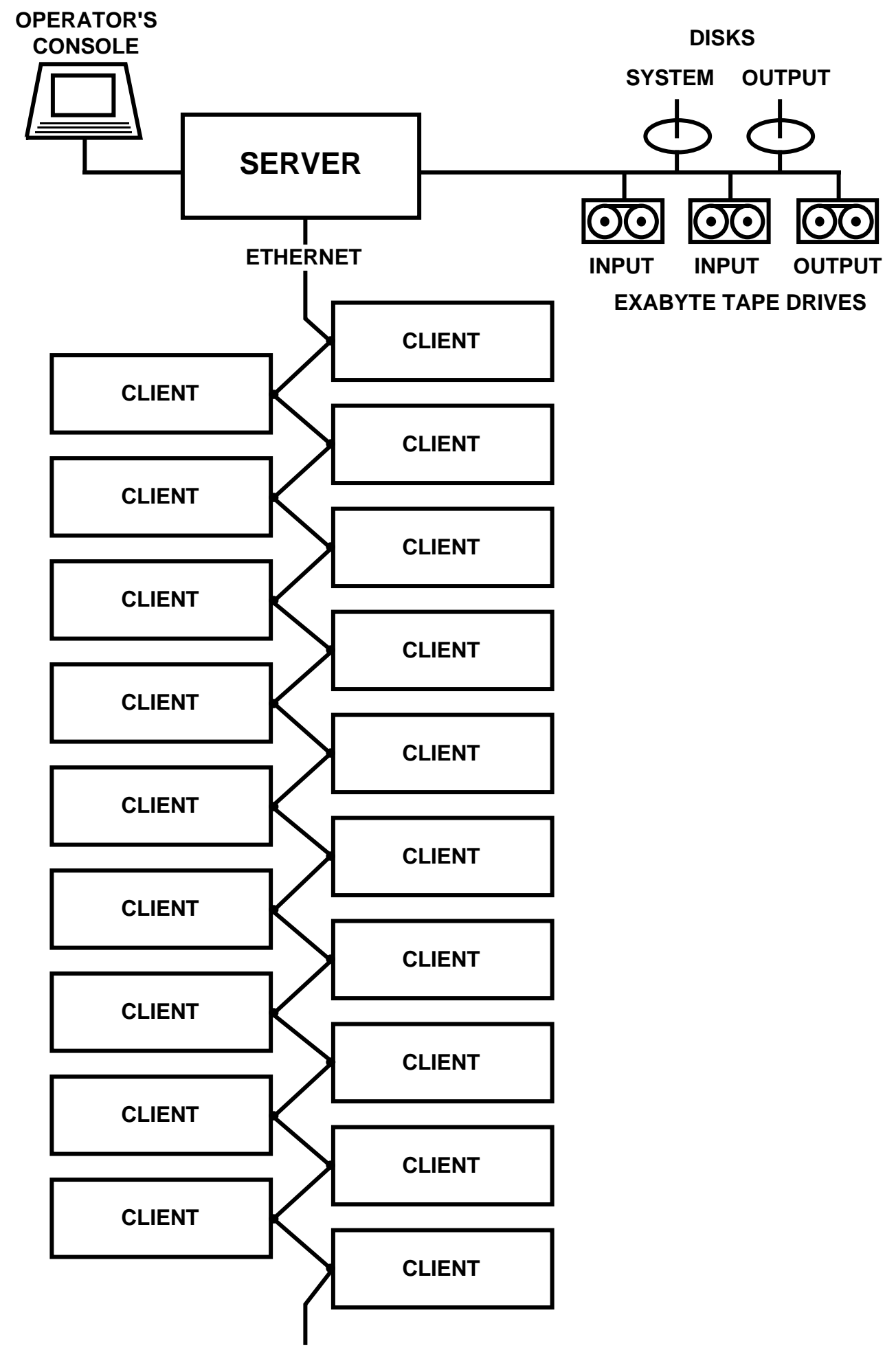

Fig. 1. Computing farm configuration at the University of Mississippi. Servers and clients are DECstation 5000 workstations running ULTRIX. Some have MIPS R3000 Processors; others have the more powerful MIPS R4000. Altogether, there are 68 processors organized into four farms, each with a separate job stream. One typical farm is shown in this diagram. The two input tape drives alternate. The output is staged through disk to tape. This I/O scheme avoids the need for continuous operator supervision. The total computing power is about 3000 mips. 
Fig. 2. A photographic overview of the University of Mississippi computing farm. Servers are on the four tables. Clients are on the racks shown as well as on desktops which are not shown. The espresso machine is on the left. 


\section{SERVER CLIENT}

MAKE_SOCKET to connect

with the clients

BIND_SOCKET to a port

so clients can find it
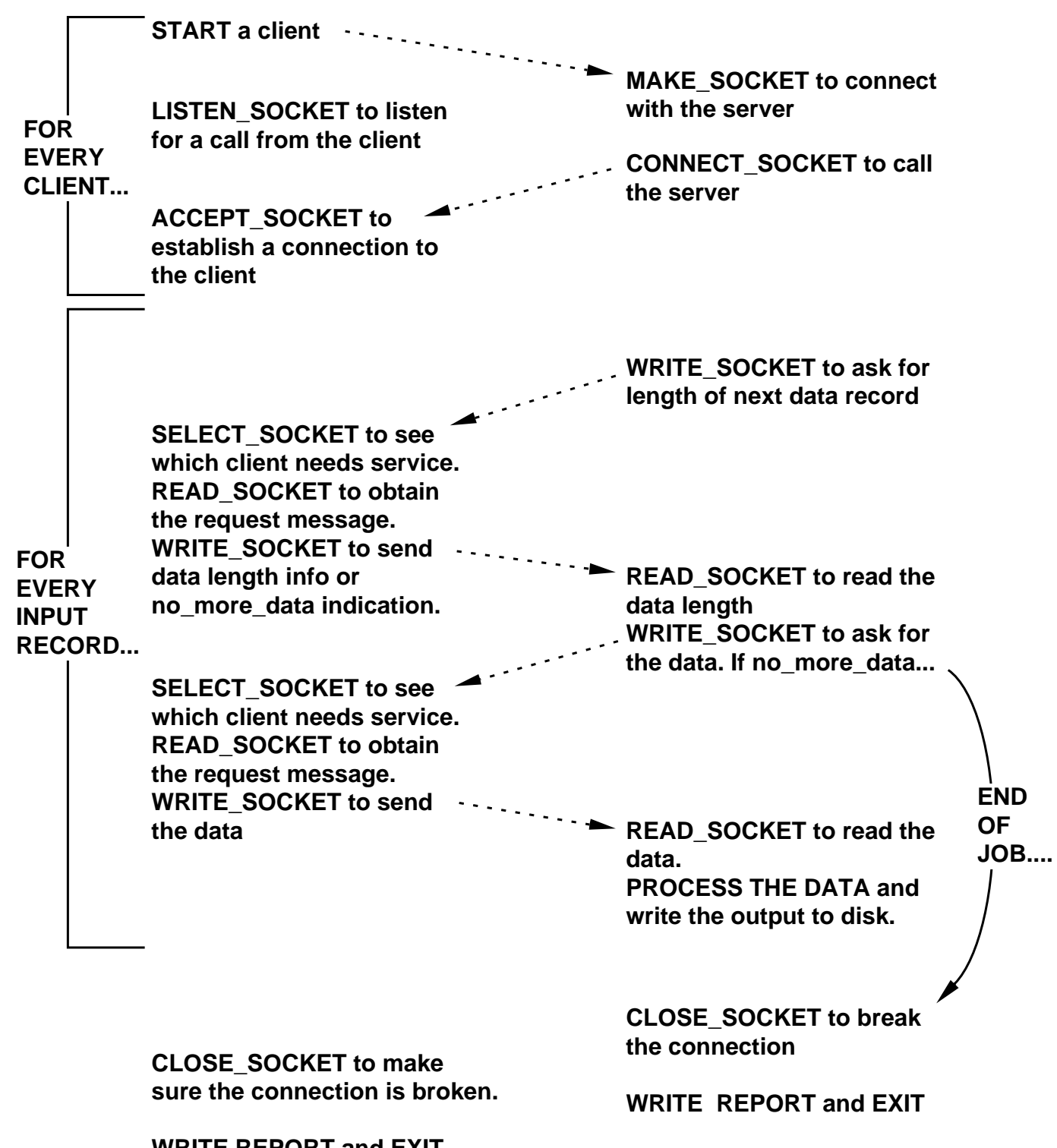

WRITE REPORT and EXIT

Fig. 3. Communication between server and clients using TCP/IP sockets. 